\title{
Anticipation boosts forgetting of voluntarily suppressed memories
}

\author{
Simon HansImayr, Philipp Leipold, and Karl-Heinz Bäuml \\ Regensburg University, Germany
}

\begin{abstract}
The role of anticipatory mechanisms in human memory control is poorly understood. Addressing the issue we investigated whether the presence of an anticipatory phase can enhance effects of cognitive control, as they occur during voluntary suppression of episodic memories. Using the Think/No-Think task, participants first learned several face-word associations, and thereafter were asked to either recall (think) or suppress (no-think) the word when provided with the word's face cue. In the one condition participants performed the Think/No-Think task in the presence of an anticipatory phase, giving participants the chance to prepare for memory suppression. In the other condition participants performed the task without such an anticipatory phase. On the final cued recall test participants were asked to recall all of the previously studied words. The results showed stronger forgetting of to-besuppressed items in the presence than absence of the anticipatory phase. The finding is first evidence for the effectiveness of anticipatory mechanisms in human memory suppression.
\end{abstract}

Keywords: Cognitive control; Anticipation; Episodic memory; Suppression; Voluntary forgetting.

Cognitive control processes can benefit from an anticipatory phase, which prepares the brain for an upcoming cognitive task. Corresponding evidence has arisen for control processes involved in task switching (Dreisbach, Haider, \& Kluwe, 2002; Lavric, Mizon, \& Monsell, 2008; Rogers \& Monsell, 1995) but to date this has not been shown in other cognitive domains; for instance, human memory. Indeed, current evidence for a role of anticipatory processes in human memory is restricted to the finding of anticipatory brain activity during memory formation (e.g., Adcock, Thangavel, Whitfield-Gabrieli, Knutson, \& Gabrieli, 2006; Otten, Quayle, Akram, Ditewig, \& Rugg, 2006). In this report we focus on anticipatory processes for memory control mechanisms, in particular processes involved in voluntary memory suppression.

In everyday life, voluntary suppression mechanisms are necessary to keep human memory free from traumatic experiences or outdated information (Bjork 1989). In the laboratory these mechanisms can be studied using the Think/No-Think (T/NT) task (Anderson \& Green, 2001; Depue, Banich, \& Curran, 2006). In this task participants are asked to either repeatedly recall or repeatedly suppress previously studied material. While repeated recall of studied material typically enhances its later memory, repeated suppression can induce later forgetting of the information (for reviews, see Bäuml, Pastötter, \& Hanslmayr, in press; Levy \& Anderson, 2002). Such suppression-induced forgetting is often assumed to be mediated by inhibitory executive-control mechanisms, which are recruited to prevent the unwanted memory from entering consciousness (Anderson \& Green, 2001; Anderson et al., 2004). According to this account, during NT trials the memory representation of the target is impaired, so that accessibility of the item is lowered regardless of which cue is provided and which retrieval route is used (for

Address correspondence to: Simon Hanslmayr, Department of Experimental Psychology, Regensburg University, 93053 Regensburg, Germany. E-mail: simon.hanslmayr@psychologie.uni-r.de 
a recent non-inhibitory account of T/NT impairment, see Tomlinson, Huber, Rieth, \& Davelaar, 2009). The finding of T/NT impairment in independent-probe tasks, i.e., tasks in which a new cue is provided at test to probe the memory item, supports this proposal (Anderson \& Green, 2001; Anderson et al., 2004; Bergström, deFockert, \& Richardson-Klavehn, 2009; but see Bulevich, Roediger, Balota, \& Butler, 2006).

Concerning the underlying neural substrates of voluntary memory suppression, fMRI studies showed that increased activity in lateral prefrontal brain regions and decreased activity in memory relevant regions (e.g., hippocampus) predicts forgetting of the suppressed information (Anderson et al., 2004; Depue, Curran, \& Banich, 2007). These findings suggest that suppressioninduced forgetting is mediated by lateral prefrontal brain areas, which exert top-down control over memory-relevant brain structures in order to inhibit memory processing. Consistent with these findings, EEG studies examining event-related potentials (ERP) during the suppression of an unwanted memory, showed a reduction in ERP components that index successful recall or recognition (Bergström, Velmans, de Fockert, \& Richardson-Klavehn, 2007; Hanslmayr, Leipold, Pastötter, \& Bäuml, 2009). For instance, Hanslmayr et al. (2009) found that a reduced sustained positivity over right frontal and left parietal electrodes, an ERP component that is believed to index recall success (Allan, Doyle, \& Rugg, 1996), predicted the amount of T/NT forgetting.
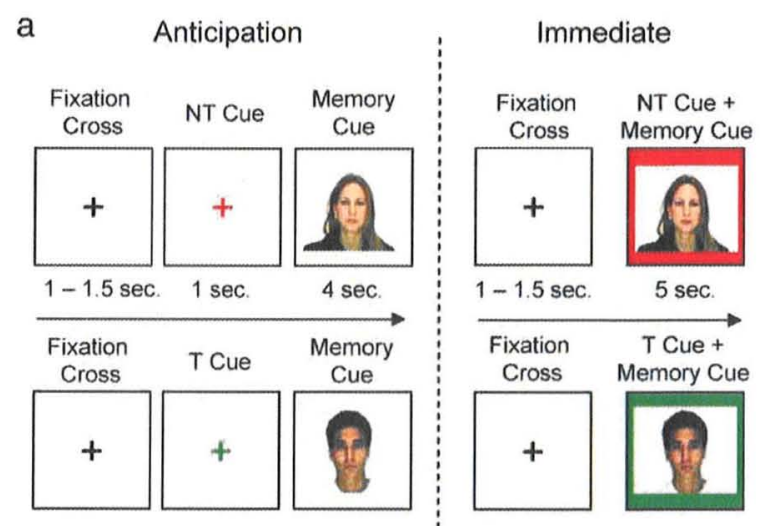

In a recent ERP study we demonstrated the existence of anticipatory brain activity in the T/NT task. When participants were allowed to prepare for suppressing previously studied material, neural preparatory activity arose that was related to later forgetting (Hanslmayr et al., 2009). These results suggest that anticipatory mechanisms prepare the brain for suppressing unwanted memories, presumably by top-down driven inhibition of memory relevant brain areas. While these results provide evidence for the existence of preparatory activity in memory control, the results bear no implication on the effectiveness of such an anticipatory phase, because the relationship between anticipatory brain activity and forgetting was only correlative in nature. In the present T/NT study we therefore directly contrasted forgetting effects in the presence versus absence of an anticipatory cue (Figure 1a). If anticipatory activity arises and is effective for memory suppression, suppressioninduced forgetting should be stronger in the presence than absence of an anticipatory cue.

\section{METHOD}

\section{Participants}

A total of 48 students (40 females) with a mean age of 21.5 years $(S D=2.99)$ participated for course credit. All volunteers were native German speakers and reported no history of neurological or psychiatric diseases.

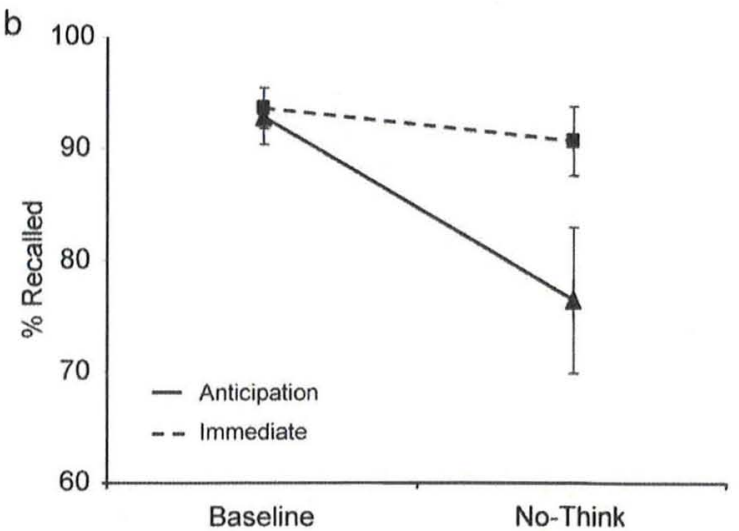

Figure 1. (a) The Think/No-Think procedure. In the Anticipation condition a coloured fixation cross (T/NT cue) predicted either a forthcoming Think (green) or a forthcoming No-Think trial (red). In the Immediate condition the memory cue was presented simultaneously with the T/NT cue, which was either a green (T) or red (NT) frame surrounding the memory cue. (b) Recall performance for Baseline and No-Think items for the Anticipation and the Immediate conditions. Error bars indicate standard errors. 


\section{Materials, design, and procedure}

The study material consisted of 54 face-word associations. The faces had a neutral emotional expression and were drawn from the AR Face Database (Martinez \& Benavente, 1998). The words were taken from different semantic categories (Battig \& Montague, 1969).

Following prior work (Depue et al., 2006; Hanslmayr et al., 2009), the experiment consisted of two blocks, each including a complete T/NT procedure (training phase, T/NT phase, and testing phase). Within each block, 27 face-word pairs served as item material, including three filler items used to familiarise participants with the procedure.

The participants were randomly assigned to one of the two experimental groups: Anticipation vs Immediate. The two groups were matched for sex (20 females in each group) and age (21.01 vs 22.04; Anticipation and Immediate group respectively). The Anticipation group performed the T/NT task in the presence of an anticipatory cue, whereas the Immediate group performed the task without such an anticipatory cue. Regarding material, of the 24 experimental face-word pairs, 8 were used as suppress items, 8 as respond items, and 8 as baseline items. The material was counterbalanced across item types and blocks.

Training phase. The phase comprised two study-test cycles. In the study trials each faceword association was presented for 5 seconds. In the test trials each face was presented for 4 seconds and the participants were asked to recall the appropriate word.

Think/No-think phase. In this phase the faces were presented as memory cues and participants were asked either to recall the associated word (T) or not to let the associated word enter consciousness (NT). During NT trials all volunteers were strongly encouraged to keep their fixation on the face and to not generate alternative associations. The sequence of respond and suppress trials was pseudo-randomised, with the restriction that the same condition was not repeated more than three times. A total of 10 repetitions of suppress and respond trials were conducted (Depue et al., 2006; Hanslmayr et al., 2009). No baseline items were presented.

For the Anticipation group, the T and NT cues were signalled by a fixation cross which turned either green (T) or red (NT) before the face was presented. The T/NT cue was shown for 1 second, and the face was presented for 4 seconds. For the Immediate group, the face was presented for 5 seconds, and was surrounded by a green $(\mathrm{T})$ or red (NT) frame indicating whether participants should recall or suppress the associated word (Figure 1a). The unequal length of presentation of the memory cue ( 4 vs 5 seconds) was necessary to keep the total time of suppression equal between the two groups ( 5 seconds).

Testing phase. In the final testing phase each face of the initially studied face-word associations was presented, and the participants were given 4 seconds to name the appropriate word. The filler items were not tested.

Compliance questionnaire. At the end of the experiment a questionnaire was used to check whether the participants had followed the NT instruction. The questionnaire contained three statements, and was a German translation of the compliance questionnaire used by Bulevich et al. (2006). The participants were asked to indicate how often, if ever, they used one of the strategies expressed in the statements (e.g., whether they silently rehearsed the NT word during the suppression trials). The ratings were given from 0 (never) to 4 (very frequently). This allowed for computing an individual compliance score ranging from 0 (total compliance) to 12 (no compliance).

\section{RESULTS}

\section{Training phase}

After the second test trial of the training phase, the mean recall rates were $80.3 \%$ for the Anticipation group and $80.7 \%$ for the Immediate group $\left(t_{46}=0.12 ; p>.5\right)$. Thus the two groups did not differ in their ability to memorise the face-word pairs. As in previous studies (Anderson et al., 2004; Bergström et al., 2009; Hanslmayr et al., 2009 ), data analysis in the test phase was based only on those face-word pairs that were remembered correctly at the end of the second studytest cycle.

\section{Test phase}

Mean recall rates and standard errors for the Anticipation and the Immediate condition are reported in Table 1. To examine the effects of 
TABLE 1

Mean recall rates and $S E$

\begin{tabular}{lccc}
\hline & Baseline & No-Think & Think \\
\hline Anticipation & $92.9(2.5)$ & $76.4(6.6)$ & $97.2(1.7)$ \\
Immediate & $93.6(1.6)$ & $90.6(3.1)$ & $96.2(2.4)$ \\
\hline
\end{tabular}

Mean recall rates (\%) and standard errors (in brackets) of Think, No-Think, and Baseline items are reported for the Anticipation and the Immediate conditions.

anticipation on suppression-induced forgetting, a two-way ANOVA with the factors of Item type (baseline vs NT) and Group (Anticipation vs Immediate) was carried out. A significant main effect of Item type was obtained $\left(F_{1,46}=8.67\right.$; $M S E=262.73 ; p<.005)$, reflecting that repeated suppression trials led to subsequent forgetting. A significant Item type by Group interaction was also obtained $\left(F_{1,46}=4.18 ; M S E=262.73 ; p<\right.$ .05 ), indicating that forgetting was stronger for the Anticipation group than the Immediate group (see Figure 1b). No significant main effect of Group arose $\left(F_{1,46}=2.75 ; M S E=486.51 ; p>.1\right)$.

To investigate beneficial effects of anticipation, a two-way ANOVA with the factors of Item type (baseline vs T) and Group was computed. No significant main effect of Item type emerged $\left(F_{1,46}=1.89 ; M S E=109.87 ; p>.15\right)$, although a tendency for above-baseline remembering was observed for $\mathrm{T}$ items. Also, no significant interaction between Item type and Group arose $\left(F_{1,46}=\right.$ $0.43 ; M S E=109.87 ; p>.5$ ), and no significant main effect of Group was obtained $\left(F_{1,46}=0.10\right.$; $M S E=111.28 ; p>.5$ ).

\section{Compliance scores}

No significant difference emerged concerning the scores obtained from the compliance questionnaire, yielding means of $4.04(S D=2.33)$ for the Anticipation and $3.38(S D=2.11)$ for the Immediate group $\left(t_{46}=1.04 ; p>3\right)$. No significant correlation between the compliance scores and amount of forgetting was obtained (Pearson's $R_{47}=0.11 ; p>.4$ ). Thus both groups followed the suppression instructions equally, and compliance had no influence on the results.

\section{Additional analysis}

In an additional step the data were analysed by taking all memory items into account, regardless
TABLE 2

Mean recall rates and SE with data of all items taken into account

\begin{tabular}{lccc}
\hline & Baseline & No-Think & Think \\
\hline Anticipation & $79.7(3.6)$ & $73.4(5.8)$ & $90.1(2.8)$ \\
Immediate & $83.3(4.1)$ & $81.8(3.7)$ & $93.2(1.8)$ \\
\hline
\end{tabular}

Mean recall rates (\%) and standard errors (in brackets) of Think, No-Think, and Baseline items are reported for the ANTICIPATION and the IMMEDIATE condition. In contrast to Table 1, recall performance for the three item types is reported when data of all items were taken into account, i.e., regardless of whether the single items were correctly remembered during the training phase or not.

of whether they were correctly remembered or not at the end of the second study-test cycle. The results of this analysis are reported in Table 2. Concerning the suppression effect, the pattern of results did not change substantially but the main effect for Item type $\left(F_{1,46}=1.24 ; M S E=366.21\right.$; $p>.25)$, as well as the Item type by Group interaction $\left(F_{1,46}=0.44 ; M S E=131.84 ; p>.5\right)$ failed to reach significance. Concerning the beneficial effect, the additional analysis revealed significantly higher recall rates for $\mathrm{T}$ in contrast to baseline items $\left(F_{1,46}=17.50 ; M S E=2475.58\right.$; $p<.001)$. However, no significant Item type by Group interaction was obtained $(F<1)$, showing that anticipation had no impact on the beneficial effects of memory retrieval (Figure 2).

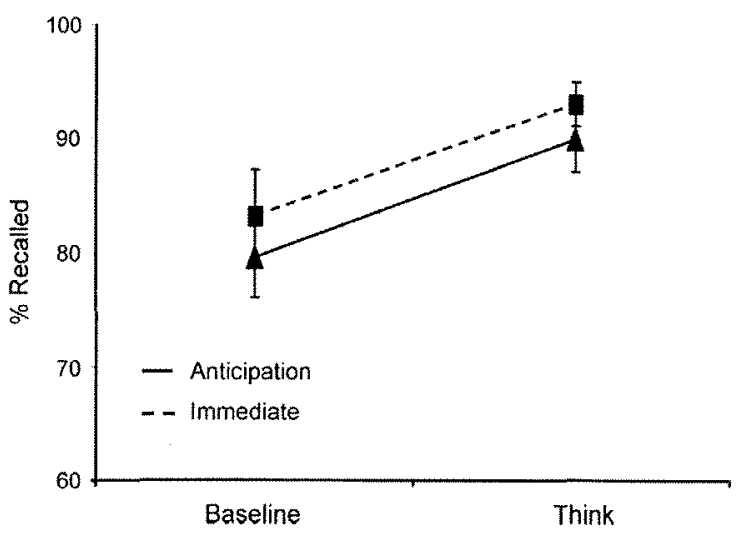

Figure 2. Recall performance for Baseline and Think items for the Anticipation and the Immediate conditions. Error bars indicate standard errors. In contrast to Figure $1 \mathrm{~b}$, recall performance for the two item types is reported when data of all items were taken into account, i.e., regardless of whether the single items were correctly remembered during the training phase or not. 


\section{DISCUSSION}

The present results demonstrate that anticipatory mechanisms boost forgetting of voluntarily suppressed memories. This finding is in line with the results of a previous study, in which it was shown that anticipatory brain mechanisms are related to forgetting in the T/NT paradigm (Hanslmayr et al., 2009). However, one shortcoming of the former study was that the relationship between anticipatory mechanisms and suppressioninduced forgetting could only be drawn on a correlative basis. This restriction left open the question of whether such anticipatory mechanisms are functionally relevant for the suppression of unwanted memories. In the present study evidence for such a functional relevance is reported by showing that forgetting is stronger when participants are given an anticipatory phase than when no such anticipatory phase is provided. This is first evidence for the effectiveness of anticipatory mechanisms in human memory suppression.

Previous fMRI studies suggest that voluntary memory suppression is mediated by the lateral prefrontal cortex, which inhibits activity in memory relevant brain structures (e.g., hippocampus; Anderson et al., 2004; Depue et al., 2007). Converging results arose in electrophysiological studies, reporting a reduction of memory relevant ERP components during the suppression of NT items (Bergström et al., 2007; Hanslmayr et al. 2009). In view of this prior work, the present results suggest that the neural machinery underlying voluntary suppression of unwanted memories is already activated by a preparatory cue, i.e., before the memory cue is presented. Such pre-activation of the network may be a highly efficient way to prepare the brain for inhibiting an upcoming memory item, thereby increasing the likelihood of its being forgotten on a later test. The results complement findings from prior memory work, which showed that anticipatory brain activity mediates memory formation (e.g. Adcock et al. 2006; Otten et al. 2006). The present findings are also in line with studies from other cognitive domains, like task switching (Dreisbach et al., 2002; Lavric et al., 2008; Rogers \& Monsell, 1995), and suggest that the beneficial effects of anticipatory mechanisms may generalise to other paradigms in which cognitive control processes are involved.
In line with previous studies (Anderson \& Green, 2001; Bulevich et al., 2006), we found that repeated retrieval has a beneficial effect on memory performance of the $\mathrm{T}$ items (see Figure 2). However, this effect was evident only when data of all items were taken into account, i.e., when analysis was not restricted to those items that were correctly remembered after the training phase. Because recall rates in the baseline condition were fairly high in the present study, restricting analysis to those items that were correctly remembered after the training phase induced a ceiling effect, which did not leave enough room to show the expected beneficial effect of retrieval. Importantly, the presence or absence of an anticipatory phase had no effect on the benefits of repeated memory retrieval. This suggests that, in the T/NT paradigm, anticipation specifically boosts memory suppression, but not memory enhancement.

Several previous studies demonstrated that the repeated suppression of memory items can lead to their later forgetting (Anderson \& Green, 2001; Depue et al., 2006; Hanslmayr et al., 2009). At the same time, however, difficulties in replicating below-baseline forgetting of NT items have been reported in the study by Bulevich et al. (2006) who utilised 16 repetitions of T/NT trials. Thus questions have been raised about the validity of the forgetting effect. Whereas one reason for not getting the forgetting effect in this task might be that the size of the effect is typically small $(<10 \%)$, there is also evidence that amount of the observed forgetting can be modulated by several factors. One such factor is the number of suppression trials, with evidence that the forgetting is absent with a small number of trials $(<5)$ but is present with a larger number of trails (>10; Anderson \& Green, 2001; Depue et al., 2006; Hanslmayr et al., 2009). A second factor is item material, with the demonstration that the forgetting is stronger for negative emotional material than for neutral material (Depue et al., 2006). The present results add to this line of studies by showing that the presence or absence of an anticipatory phase is another such factor that can modulate suppression-induced forgetting.

At first glance, our results appear inconsistent with those reported by Bulevich et al. (2006), who failed to show below baseline forgetting in two experiments despite the use of an anticipatory phase. In contrast to the present study, however, Bulevich et al. did not restrict data analysis to 
those items that were correctly remembered after the training phase. Instead, data of all items were taken into account, regardless of whether the participants were able to memorise the respective item after training or not. We replicated Bulevich et al. (2006) when analysing the data of all items (Table 2). Arguably, however, restricting analysis to those items that were correctly remembered after the training phase should be more sensitive to detecting the (weak) suppression effect than analysing the data of all items. Consistently, several recent T/NT studies analysed their behavioural data conditionalised on study performance after the training phase (Anderson et al., 2004; Bergström et al., 2009; Hansimayr et al., 2009). Following this procedure, we found evidence that anticipation boosts memory suppression in the T/NT paradigm.

Taken together, the present results demonstrate that an anticipatory phase, prior to the presentation of a memory cue, enhances forgetting of voluntarily suppressed information. This finding shows, for the first time, that preparatory mechanisms are effective for the suppression of contents in episodic memory. Together with our previous study (Hanslmayr et al., 2009) this finding suggests that the brain pre-activates cognitive control networks. Such a pre-activation might prevent a suppressed memory item from entering consciousness, thereby decreasing its likelihood of being remembered in the future.

\section{REFERENCES}

Adcock, R. A., Thangavel, A., Whitfield-Gabrieli, S., Knutson, B., \& Gabrieli, J. D. E. (2006). Rewardmotivated learning: Mesolimbic activation precedes memory formation. Neuron, 59, 507-517.

Allan, K., Doyle, M. C., \& Rugg, M. D. (1996). An event-related potential study of word-stem cued recall. Cognitive Brain Research, 4, 251-262.

Anderson, M. C., \& Green, C. (2001). Suppressing unwanted memories by executive control. Nature, 410, 366-369.

Anderson, M. C., Ochsner, K. N., Kuhl, B., Cooper, J., Robertson, E., Gabrieli, S. W., et al. (2004). Neural systems underlying the suppression of unwanted memories. Science, 303, 232-235.

Battig, W. F., \& Montague, W. E. (1969). Category norms for verbal items in 56 categories: A replication and extension of the Connecticut category norms. Journal of Experimental Psychological Monographs, 80, 1-64.

Bäuml, K. H., Pastötter, B., \& Hanslmayr, S. (in press). Binding and inhibition in episodic memory cognitive, emotional, and neural processes. Neuroscience and Biobehavioral Reviews.

Bergström, Z., de Fockert, J., \& Richardson-Klavehn, A. (2009). ERP and behavioral evidence for direct suppression of unwanted memories. Neuroimage, 48 , 726-737.

Bergström, Z., Velmans, M., de Fockert, J., \& Richardson-Klavehn, A. (2007). ERP evidence for successful voluntary avoidance of conscious recollection. Brain Research, 1151, 119-133.

Bjork, R. A. (1989). Retrieval inhibition as an adaptive mechanism in human encoding. In $H$. L. Roediger III \& F. I. M. Craik (Eds.), Varieties of memory and consciousness: Essays in honour of Endel Tulving (pp. 309-330). Hillsdale, NJ: Lawrence Erlbaum Associates Inc.

Bulevich, J. B., Roediger, H. L. III, Balota, D. A., \& Butler, A. C. (2006). Failures to find suppression of episodic memories in the think/no-think paradigm. Memory and Cognition, 34, 1569-1577.

Depue, B. E., Banich, M. T., \& Curran, T. (2006). Suppression of emotional and non-emotional content in memory. Psychological Science, 17, 441-447.

Depue, B. E., Curran, T., \& Banich, M. T. (2007). Prefrontal regions orchestrate suppression of emotional memories via a two-phase process. Science, 317, 215-219.

Dreisbach, G., Haider, H., \& Kluwe, R. H. (2002). Preparatory processes in the task-switching paradigm: Evidence from the use of probability cues. Journal of Experimental Psychology: Learning, Memory and Cognition, 28, 468-483.

Hanslmayr, S., Leipold, P., Pastötter, B., \& Bäuml, K-H. (2009). Anticipatory signatures of voluntary memory suppression. Journal of Neuroscience, 29, 2742-2747.

Lavric, A., Mizon, G. A., \& Monsell, S. (2008). Neurophysiological signature of effective anticipatory task-set control: A task-switching investigation. European Journal of Neuroscience, 28, 1016-1029.

Levy, B. J., \& Anderson, M. C. (2002). Inhibitory processes and the control of memory retrieval. Trends in Cognitive Sciences, 6, 299-305.

Martinez, A. M. \& Benavente, R. (1998). The AR face database. CVC Technical Report, 24(June).

Otten, L. J., Quayle, A. H., Akram, S., Ditewig, T. A., \& Rugg, M. D. (2006). Brain activity before an event predicts later recollection. Nature Neuroscience, 9 , 489-491.

Rogers, R. D., \& Monsell, S. (1995). The cost of a predictable switch between simple cognitive tasks. Journal of Experimental Psychology: General, 124, 207-231.

Tomlinson, T. D, Huber, D. E., Rieth, C. A., \& Davelaar, E. J. (2009). An interference account of cue-independent forgetting in the no-think paradigm. Proceedings of the National Academy of Sciences USA, 106, 15588-15593. 